\title{
Entre texte et interaction : propositions méthodologiques pour une approche discursive de l'argumentation en sciences du langage
}

\author{
Jacquin, Jérôme \& Micheli, Raphaël \\ Université de Lausanne \\ jerome.jacquin@unil.ch \& raphael.micheli@unil.ch
}

\begin{abstract}
Si l'on considère l'abondance et la diversité des travaux qui, dans le champ des sciences du langage, se proposent d'éclairer le fonctionnement de l'argumentation - que ce phénomène soit localisé au niveau de la langue, du texte, du discours ou de l'interaction -, on admettra sans doute aisément que la notion même d'«argumentation » est polysémique. Les diverses approches octroient, de manière explicite ou implicite, un sens particulier à cette notion, en fonction des données langagières étudiées et des objectifs théoriques poursuivis. Une telle situation s'accompagne, pour le chercheur, de l'exigence méthodologique suivante, bien résumée par Marianne Doury: "L'éclatement disciplinaire et théorique des recherches en argumentation impose de préciser, avant toute analyse, à quelle conception de l'argumentation on se réfère » $(2003: 11)$. Nous menons depuis plusieurs années, à titre individuel ou collectif, des recherches empiriques sur le fonctionnement de l'argumentation dans divers genres du discours politique, et cela à partir de données langagières variées (de l'oral polygéré type "débat » à l'écrit monogéré type «article de presse » en passant par l'oral monogéré type «allocution») ${ }^{1}$. Ces travaux nous ont progressivement conduits à tenter d'expliciter et de systématiser une conception de l'argumentation, dont nous voudrions idéalement qu'elle allie la rigueur théorique (en ce qu'elle prend la peine d'expliciter ses décisions conceptuelles et de les référer, le cas échéant, aux travaux d'autres chercheurs) et l'adéquation empirique (en ce qu'elle permet d'outiller la description de pratiques langagières variées). L'enjeu du présent article est de formuler, de justifier et de mettre en discussion cette conception de l'argumentation : comme nous tenterons de le montrer, l'originalité de celle-ci tient à ce qu'elle se situe à la croisée de la linguistique textuelle, de l'analyse du discours et de l'analyse des interactions, et qu'elle vise à intégrer de façon cohérente des savoirs relatifs à ces trois domaines connexes².
\end{abstract}

Les diverses théorisations de l'argumentation dans le cadre des sciences du langage francophones tendent, très schématiquement, à se concentrer sur deux composants majeurs, que nous appellerons - par souci de clarification du débat ${ }^{3}$ - les composants textuel et discursif-interactionnel. Sans prétendre faire un "état de l'art» détaillé, on peut ici, sur la base de quelques travaux qui revendiquent explicitement le fait de contribuer à une théorie de l'argumentation (et pas seulement à des analyses ponctuelles de phénomènes argumentatifs), esquisser la manière dont ces composants sont envisagés. Mettre l'accent sur le composant textuel, c'est faire l'hypothèse que l'argumentation est d'abord reconnaissable - par les locuteurs ordinaires comme par le chercheur - grâce au mode spécifique d'agencement des énoncés qu'elle engage dans la matérialité d'un texte. On pense ici aux travaux qui, dans le cadre de la linguistique textuelle, décrivent les formes de séquentialité qu'implique l'argumentation (Adam 2004, 2011), notamment à partir d'une reprise du schéma classique de Stephen Toulmin (1958). De manière plus large, c'est la question textuelle de l'étayage, que l'on peut aussi travailler à partir de l'étude microlinguistique des connecteurs (tradition richement représentée dans l'espace francophone) ou encore des types d'arguments (tradition pour l'heure majoritairement représentée dans la recherche anglophone consacrée aux « argumentation schemes", voir Walton, Reed \& Macagno 2008). Mettre l'accent sur le composant discursif-interactionnel, c'est faire l'hypothèse que l'argumentation est d'abord reconnaissable du fait qu'elle constitue un certain type d'activité verbale : l'idée est que cette activité doit pouvoir être aussi bien caractérisée dans sa plus grande généralité (selon quels rôles implique-t-elle typiquement les locuteurs? Vers quel type de visées ces locuteurs s'orientent-ils ?) que dans la diversité de ses réalisations au sein de situations et de genres de discours spécifiques. Dans l'espace francophone, ce composant discursif-interactionnel est au centre d'importants travaux qui tentent de penser 
l'inscription de l'analyse argumentative dans le champ de l'analyse du discours (notamment Amossy 2010 [2000]) et/ou dans celui de l'analyse des interactions (Plantin 1996a; Doury 2003 ; Plantin 2005).

Dans un tel contexte, notre propos sera ici de remettre en question une double division du travail qui tend implicitement à s'instaurer selon le type de données langagières que privilégient les chercheurs et selon leurs options méthodologiques. (i) On observe, d'une part, une tendance à dissocier les composants textuel et discursif-interactionnel: l'étude du caractère séquentiel de l'argumentation prise en tant que texte se fait parfois au détriment d'une réflexion sur l'argumentation prise en tant qu'activité verbale; la réciproque est par ailleurs vraie : si l'on tente prioritairement de saisir l'argumentation comme une forme d'activité verbale, on tend alors parfois à s'éloigner d'une réflexion fine sur la textualité, ses unités et ses niveaux. (ii) On observe, d'autre part, en ce qui concerne le composant que nous appelons ici discursifinteractionnel, une tendance à dissocier le discursif de l'interactionnel. On tend soit à privilégier des données scripturales asynchrones et à s'intéresser prioritairement au fonctionnement "dialogique » du discours argumentatif (comment le locuteur représente-t-il d'autres discours au sein de son propre discours?), soit à privilégier des données orales synchrones et à s'intéresser prioritairement au fonctionnement «dialogal» du discours argumentatif (comment le locuteur construit-il sa position en réponse aux discours d'autres locuteurs avec qui il interagit et qui sont en mesure de l'interrompre et de s'opposer directement à lui ?). Or l'argumentation nous semble constituer l'exemple typique d'un objet dont la compréhension gagne très nettement à ce que l'on ne reproduise pas de façon automatique certains découpages disciplinaires (scientifiques et institutionnels) ayant cours en sciences du langage, notamment entre les approches textuelles, discursives et interactionnelles: il convient au contraire de faire l'effort d'intégrer en une démarche cohérente et explicite des données, des catégories et des pratiques d'analyse issues de ces différentes approches. Nous voudrions ainsi contribuer à l'élaboration d'une conception de l'argumentation dont la pertinence ne dépende pas exclusivement d'un (et d'un seul) type de données langagières et d'une affiliation à un (et un seul) courant des sciences du langage. L'objectif du présent article est de formuler une série de propositions méthodologiques allant dans ce sens : il s'agit d'oeuvrer à une articulation aussi rigoureuse que possible entre les composants textuel et discursif-interactionnel de l'argumentation et, pour ce dernier composant, à un traitement davantage unifié des dimensions discursive et interactionnelle.

Si nous souhaitons apporter une contribution spécifique au débat sur l'analyse de l'argumentation en sciences du langage, nous aimerions aussi, à un niveau plus général, que l'argumentation apparaisse comme un exemple à partir duquel dégager quelques-unes des «imbrications théoriques des champs de l'analyse du discours, de la pragmatique et de l'interaction » : en cela, nos propositions méthodologiques sur l'argumentation s'inscrivent, nous semble-t-il, au coeur de la thématique «Discours, pragmatique et interaction ».

Pour présenter notre démarche d'une manière aussi claire que possible et pour accroître la possibilité qu'elle soit mise en discussion, nous prenons ici le risque d'avancer une définition de travail ${ }^{4}$ de l'argumentation :

L'argumentation peut être saisie comme un mode spécifique (1) de traitement verbal du désaccord (2), qui consiste en la construction de positions (3) solides, c'est-à-dire étayées par un travail textuel de justification (4) et situées par un travail interactionnel et dialogique de positionnement (5)

Nous gloserons un à un les termes-clés de cette définition de travail ${ }^{5}$, en tâchant à chaque fois de situer notre propre démarche par rapport aux principales avancées de la recherche consacrée à l'argumentation dans le champ des sciences du langage.

\section{Une conception spécifiante de l'argumentation}

Dire de l'argumentation qu'il s'agit d'un mode spécifique de traitement verbal du désaccord, c'est prendre position sur une question centrale, souvent formulée par les chercheurs en sciences du langage ${ }^{6}$, et qui concerne l'extension qu'il convient de donner à la notion d'argumentation. 
À ce titre, si l'on considère la recherche francophone actuelle, on note l'influence de modèles théoriques qui promeuvent une conception résolument généralisante de l'argumentation. On ne s'arrêtera pas ici sur la théorie de l'argumentation dans la langue d'Anscombre et Ducrot (1988) et sur son prolongement dans les travaux récents de Ducrot (2004) et Carel (2011), dans la mesure où elle refuse explicitement de voir dans l'argumentation une activité verbale orientée vers la réalisation d'un but : cette théorie soutient, on le sait, que l'argumentation est une composante fondamentale du sens des énoncés, voire des unités lexicales - on a ici un argumentativisme généralisé (tout énoncé, tout mot « argumente » dans la mesure même où il signifie). Plus proches de nous, certaines approches discursives véhiculent également - mais selon d'autres modalités - une conception extrêmement large de l'argumentation, qui tend à faire de celle-ci une composante intrinsèque de la discursivité en général. C'est le cas de la théorie de l'argumentation dans le discours, que développe Amossy depuis une quinzaine d'années, pour laquelle «l'argumentation n'est pas un type de discours parmi d'autres : elle fait partie intégrante du discours comme tel » (2010 [2000] : 221). Même si elle concède qu'il existe bien des degrés d'argumentativité variables selon les discours (et qu'Amossy saisit au moyen du couple visée vs dimension argumentative), cette approche nous semble critiquable, car excessivement généralisante. D'une part, sur le plan pragmatique, elle base sa définition de l'argumentation sur une liste de buts dont certains sont très peu spécifiques (par exemple « orienter [les] façons de voir [de l'allocutaire] » (2010 [2000] : 36), « avoir un poids et une influence sur l'allocutaire » (2010 [2000] : 221), etc.). D'autre part, sur un plan davantage textuel, elle ne pose pas de contrainte séquentielle dans sa définition de l'argumentation : elle ne lie pas la tentative de réalisation des buts à une manière (relativement) spécifique d'agencer les énoncés.

À la différence des approches qui viennent d'être citées, la position que nous défendons ici vise, suivant notamment les travaux de Plantin ${ }^{7}$, à « localiser » l'argumentation dans le territoire de la discursivité. Il nous semble méthodologiquement peu productif de faire de l'" argumentation » une notion englobante, sous laquelle on choisit de subsumer presque tous les phénomènes pragmatiques ayant trait à la capacité du langage à exercer des effets sur autrui (des plus généraux comme agir sur lui, l'influencer, aux plus spécifiques comme le faire adhérer à une thèse). Comment, alors, saisir cette spécificité du registre argumentatif? Il nous semble qu'il faut à la fois identifier des visées relativement spécifiques qui définissent, à un niveau général, l'argumentation en tant qu'activité verbale (actualisée ensuite dans le cadre de genres de discours très variés) et identifier les corrélats de ces visées en termes de matérialité langagière.

\section{L'argumentation comme mode spécifique de traitement du désaccord}

Le fait d'associer l'argumentation avec le désaccord n'a, en soi, rien d'original. Il est même tout à fait consensuel d'affirmer que l'existence - effective ou plausible - d'un désaccord est une condition d'émergence de l'activité argumentative: on ne s'engagera pas dans une argumentation s'il l'on ne considère pas qu'il puisse exister au moins deux réponses différentes à une question donnée. Cette idée générale traverse autant la tradition rhétorique que les théories normatives contemporaines inspirées de la logique et de la dialectique. Au premier chapitre de la Rhétorique, Aristote affirme que «nous ne délibérons que sur les questions qui sont manifestement susceptibles de recevoir deux solutions opposées » (Rhét., I, 2, 1356b). Du côté des approches normatives, notamment la logique informelle et la pragma-dialectique, on fait aussi du « disagreement» ou de la « difference of opinions » les déclencheurs de l'argumentation: "Arguments are found where there is some [...] disagreement about a subject», résume Govier (1997: 1, nous soulignons).

Si l'importance du désaccord initial est unanimement soulignée, la plupart des théories franchissent toutefois un pas supplémentaire : elles posent que l'argumentation part du désaccord, mais vise en fin de compte à résorber celui-ci. On viserait, lorsqu'on argumente, à produire un certain effet sur l'allocutaire, et cet effet consisterait en un changement d'attitude de l'allocutaire par rapport à un point de vue. Bien sûr, ce changement d'attitude peut se décliner de diverses manières : conversion d'un désaccord initial en accord («persuader» et «convaincre» au sens fort) ou renforcement d'un accord déjà existant ( «accroître l'adhésion des esprits à une thèse », selon la fameuse expression de Perelman \& OlbrechtsTyteca $1970: 5$ ). Toutefois, l'idée générale selon laquelle l'argumentation implique, chez les participants, 
une visée de résorption du désaccord traverse de très nombreuses théories - qui, sur d'autres points, divergent fortement ${ }^{8}$.

Il nous semble que cette visée de résorption du désaccord, si elle est clairement associée à de nombreux genres de discours dans lesquels l'argumentation trouve à se développer, n'a pas à entrer dans la définition générale de celle-ci. En d'autres termes, l'argumentation est certes un mode de traitement parmi d'autres du désaccord ${ }^{9}$, mais sa spécificité ne réside pas selon nous dans le fait qu'elle vise à le réduire, en «persuadant»/«convainquant» l'allocutaire ou en «accroi[ssant son] adhésion ». Où convient-il alors de rechercher cette spécificité ? L'idée est que dans l'argumentation, on observe d'abord une «cristallisation ${ }^{10} \mathrm{du}$ désaccord : une question donne lieu à au moins deux réponses divergentes, dont aucune ne semble vouloir s'aligner sur l'autre. Si le désaccord se cristallise, c'est que ces réponses, d'une façon ou d'une autre, se maintiennent et que les participants ne passent pas à autre chose. C'est le deuxième point essentiel, que nous allons développer dans le reste de cet article : les réponses divergentes vont passer du simple statut de « réponse » à celui - différent - de position (voir point suivant), dans le sens où elles font l'objet d'une construction.

Pour saisir ce que l'argumentation a de spécifique eu égard au traitement du désaccord, on n'a donc, selon nous, pas besoin d'évoquer une visée de résorption du désaccord: cette visée nous semble découler des normes régissant certains genres de discours dans lesquels l'argumentation se déploie, bien plus que de l'argumentation per se (voir Doury 2003: 11 pour un point de vue similaire). Les notions cruciales nous semblent être la cristallisation du désaccord et la construction de positions qui en résulte, par un double travail d'étayage et de positionnement observable dans la matérialité langagière.

\section{La position comme notion centrale du dispositif}

Un désaccord quant aux réponses à donner à une même question (par exemple « Faut-il interdire le voile dans l'espace public ? ») constitue ainsi la condition d'émergence d'une situation argumentative faisant passer les réponses données (« oui absolument» vs « non jamais de la vie ») au statut de positions, c'està-dire de thèses soutenues par des arguments (par exemple «en vertu de la laïcité » vs « en vertu de la liberté confessionnelle»). On saisit davantage la centralité de la notion de position en prenant en considération la dimension spatiale que revêtent respectivement les expressions " occuper » et " tenir une position ». Par la première, on entend le fait que la construction d'une position implique un travail relationnel, assimilable à une localisation topographique dans un espace où d'autres positions coexistent $^{11}$. Par la seconde, on envisage la dynamique de consolidation qu'une telle construction implique, c'est-à-dire le travail de fortification venant assurer la solidité de la place forte bâtie et du territoire occupé ${ }^{12}$.

En plaçant la notion de position au centre du dispositif, on rappelle ainsi notre refus de définir l'argumentation par des visées externes ( convaincre » l'allocutaire, le «persuader », " accroître [son] adhésion », oeuvrer à la résorption du désaccord) : celles-ci ne nous semblent pas aptes à fonctionner comme dénominateur commun des multiples usages de l'argumentation que l'on peut observer empiriquement. En lieu et place de ces visées externes, il s'agit de saisir l'argumentation au travers de ses visées internes, liées à l'expérience même du désaccord et à la construction de positions dont la localisation et la fortification sont relativement indépendantes d'un potentiel effet d'attraction : il n'est bien sûr pas impossible que l'argumentation persuade, mais ceci ne peut être considéré comme définitoire.

Bien que parlantes, les métaphores spatiales de la localisation et de la consolidation restent encore abstraites et donc insuffisantes pour saisir l'argumentation dans la matérialité langagière, identifier les différentes ressources qui participent à son inscription et garantir ainsi son observabilité. Le passage par le travail de Marc Angenot, analyste du discours argumenté dans une perspective à la fois sociale et historique, nous apparaît profitable. Se débarrassant du « fardeau » de la persuasion, Angenot propose une analyse discursive de l'argumentation, attentive à ses logiques internes et fournissant du même coup une base méthodologique pour l'observation empirique des deux opérations engagées dans la construction des positions : 


\begin{abstract}
Les humains argumentent et débattent, ils échangent des « raisons » pour deux motifs immédiats, logiquement antérieurs à l'espoir, raisonnable, mince ou nul, de persuader leur interlocuteur : ils argumentent pour se justifier, pour se procurer face au monde une justification [...] inséparable d'un avoir-raison - et ils argumentent pour se situer par rapport aux raisons des autres en testant la cohérence et la force qu'ils imputent à leurs positions, pour se positionner (avec les leurs éventuellement) et, selon la métaphore polémique, pour soutenir ces positions et se mettre en mesure de résister. (2008: 441)
\end{abstract}

Engagée dans deux directions - le positionnement (la dynamique centrifuge à laquelle nous référions par le terme de «localisation») et la justification (la dynamique centripète de la «consolidation ») -, la construction argumentative des positions conduit l'analyste à se situer à une intersection disciplinaire intéressante. D'une part, la justification nous fait entrer dans la problématique de l'étayage. Comme on le verra, le sujet argumentant entend assurer la solidité de sa position en articulant à la formulation de la thèse un certain nombre d'énoncés-arguments en soutien. C'est le versant davantage textuel de l'argumentation, relatif à la dimension séquentielle (linéarisation) et hiérarchique (empaquetage) de la matérialité discursive. D'autre part, le positionnement nous conduit à considérer l'argumentation d'un point de vue davantage discursif et interactionnel, relatif à l'expérience du désaccord comme mode de gestion de la co-présence et aux ressources, dialogiques et polyphoniques, de représentation du discours et du point de vue d'autrui.

\title{
4 Le travail textuel d'étayage
}

La conception que nous défendons ici est, on l'a dit, spécifiante : l'argumentation est inséparable d'un mode relativement spécifique d'agencement des énoncés, d'une forme de séquentialité. Dans une optique de linguistique textuelle, attentive aux unités intermédiaires de structuration entre l'énoncé et le texte, l'analyse de l'argumentation doit pouvoir saisir celle-ci comme un "regroupement typé » d'énoncés (selon l'expression d'Adam 2011: 23). Bien sûr, notre approche ne se réduit pas à cette dimension textuelle (on verra plus loin l'importance accordée à la dimension dialogique et interactionnelle relative à l'articulation du discours et du contre-discours), mais elle en (ré)affirme le caractère incontournable.

L'idée que l'argumentation se traduit, textuellement parlant, par une manière spécifique d'agencer les énoncés doit être quelque peu précisée. Pour que l'on puisse parler d'argumentation, il faut minimalement - que l'on observe un certain type de relation entre les énoncés, qui assigne à ceux-ci des statuts distincts. Dans les travaux anglophones, la relation entre les énoncés est principalement saisie par les verbes «to support» et « to justify »; dans la recherche francophone, on parle de «justifier » ou - ce sera le terme retenu ici - d'« étayer » un énoncé par un autre (ou par d'autres) énoncé(s). La désignation des statuts qui se voient assignés aux différents énoncés dans le cadre de la «relation argumentative » (Plantin 1996a : 14) ne fait pas l'objet d'un consensus terminologique : pour l'énoncé étayé, on parle le plus souvent - en français - de la «conclusion » ou de la «thèse » et - en anglais - du «claim »; pour les énoncés étayants, on parle des «arguments », des "prémisses» ou des «raisons ». A cela s'ajoute l'idée que la relation d'étayage est assurée par un énoncé souvent implicite qui garantit le lien entre les prémisses et la conclusion : c'est, on le sait, le « warrant» du schéma de Toulmin ou, dans la majorité des approches francophones inspirées de la rhétorique, le topos. Que l'argumentation implique minimalement un réseau d'énoncés dans lequel on peut observer une relation d'étayage assignant aux énoncés les statuts interdépendants de prémisse(s) et de conclusion, c'est là une idée qui est très largement répandue. Il peut paraître oiseux de la rappeler ici. En effet, dans les approches anglophones relevant de la logique informelle et de la pragma-dialectique, elle est constamment réaffirmée : "In the first instance, an argument appears as a premise-conclusion structure : Reasons are produced to justify a target proposition, which is the conclusion», affirme Johnson (2000: 160), tandis que Van Eemeren et Grootendorst évoquent une «constellation of propositions justifiying [...] the proposition expressed in the standpoint » (2004 : 1). Il nous semble, en revanche, que les choses sont plus ambiguës dans la recherche francophone sur l'argumentation. Certes, les travaux de linguistique textuelle insistent sur la spécificité séquentielle de l'argumentation (par rapport aux autres types de séquence : narration, description, explication et dialogue, 
voir Adam 2011), et Plantin, commentant la reprise du schéma de Toulmin et la triade [Donnée/Loi de passage/Conclusion], parle dès 1990 d'une «"cellule argumentative", articulée aux dimensions d'un texte » (1990: 33). Toutefois, certaines approches généralisantes tendent à ne plus intégrer une quelconque contrainte séquentielle dans leur définition : l'argumentation ne s'accompagne plus forcément d'un travail textuel d'étayage, observable dans la matérialité langagière. C'est le cas notamment, nous semble-t-il, de la théorie de l'« argumentation dans le discours » (Amossy 2010 [2000]) et de la définition de l'argumentation comme schématisation dans les travaux de Jean-Blaise Grize : si la schématisation est «l'élaboration, par le moyen de la langue, d'un micro-univers que A présente à $\mathrm{B}$ dans l'intention d'obtenir un certain effet sur lui » (1982 : 188), elle n'implique pas forcément de relation d'étayage entre les énoncés.

Notre position est qu'une approche langagière de l'argumentation ne peut faire l'impasse sur le travail textuel d'étayage des énoncés. Bien sûr, cette dimension ne suffit pas à elle seule à caractériser de façon adéquate le discours argumentatif, mais elle participe de façon tout à fait décisive à son accountability son caractère « reconnaissable », non seulement par le chercheur, mais aussi, et de manière cruciale, par les locuteurs ordinaires. Que l'on pense aux «représentations ordinaires de l'argumentation » (Doury 2003) et aux commentaires méta-argumentatifs produits par les locuteurs: il paraît probable que la reconnaissance d'une relation d'étayage participe de façon significative à la catégorisation d'un segment textuel comme relevant de l'argumentation. Ce point apparaît d'autant plus clairement dans les commentaires méta-argumentatifs négatifs qui, sur la base d'une absence d'étayage, refusent d'octroyer à un discours le statut d'argumentation. On réaffirme donc ici la nécessité d'un composant textuel d'étayage dans la définition générale de l'argumentation, et cela vaut - il faut le souligner - autant pour les données langagières orales qu'écrites. Il est vrai que les catégories descriptives dont on dispose pour saisir l'argumentation comme mode spécifique d'agencement des énoncés - le schéma de l'argumentation de Toulmin, la séquence argumentative et la typologie des arguments selon la nature du rapport entre les prémisses et la conclusion - ont été principalement élaborées à partir de et testées sur des données écrites : la réflexion doit à présent, nous semble-t-il, également embrasser le champ des productions orales, en tenant compte des problèmes spécifiques de segmentation de la chaîne verbale qu'il pose (si l'argumentation peut être partiellement saisie comme une "composition d'énoncés » ou une « suite de propositions », quelle est la pertinence d'unités comme les « énoncés » et les «propositions » à l'oral ?).

On voit, de manière générale, que l'observabilité de ce composant d'étayage dans la matérialité textuelle reste extrêmement difficile lorsqu'on s'occupe de décrire des données langagières attestées. C'est là un problème méthodologique que nous ne pouvons aborder ici dans toute sa complexité; nous nous contenterons de soulever rapidement quelques questions qui nous paraissent figurer à l'agenda d'une approche de l'argumentation qui prend au sérieux cette question textuelle de l'étayage : il ne s'agit donc pas de donner un « mode d'emploi », mais de soulever trois questions centrales relatives à l'applicabilité des catégories descriptives traditionnellement utilisées lors de l'analyse argumentative des textes.

(i) Il y a d'abord la question de la correspondance entre les unités argumentatives, d'une part, et les unités linguistiques, d'autre part. L'une des difficultés d'une approche langagière de l'argumentation est qu'il n'est absolument pas évident de savoir à quel type exact d'unités linguistiques on a affaire lorsque l'on parle de « prémisses », de « conclusions », de « lois de passage »... Dans l' « architecture » des faits langagiers, on a tendance à y voir des unités de rang phrastique, mais ce n'est là qu'une des possibilités. Comme le suggère Atayan (2009), les unités argumentatives traditionnellement envisagées peuvent parfois correspondre, dans la matérialité langagière, à des unités infra-phrastiques (proposition subordonnée, apposition nominale, épithète détachée...). Le cas inverse doit aussi être envisagé : les unités fonctionnelles du schéma de l'argumentation peuvent correspondre à des unités supra-phrastiques (groupe de phrases, paragraphes...). Comme le pressentait déjà Plantin dans ses Essais sur l'argumentation (1990 : 33-34) en évoquant l'«applicabilité » du schéma de Toulmin pour l'analyse textuelle, la «cellule argumentative » ne «dépend pas d'une forme ou d'un élément textuel déterminé ; elle est aussi bien maquette que modèle réduit, elle correspond aussi bien à un énoncé qu'à un paragraphe ». 
(ii) La question de la linéarité est elle aussi complexe. Comme l'ont noté plusieurs auteurs (notamment Grize 1996 : 16-17 ; Adam 2004 : 93, 2011 : 134), un schéma comme celui de Toulmin ne préjuge pas de l'ordre dans lequel les différents composants vont apparaître dans la matérialité textuelle. On observe, à ce titre, deux ordres séquentiels majeurs : un ordre progressif, selon lequel on passe des prémisses à la conclusion, et un ordre régressif, selon lequel on part de la thèse et l'on fournit ensuite les raisons censées la justifier. Dans tous les cas, une approche de l'argumentation attentive à la question textuelle de l'étayage doit selon nous affronter cette question de la linéarité. S'ajoute à cette question de la linéarité la possible absence matérielle de l'un ou l'autre des composants du schéma de l'argumentation - c'est la question de l'implicite. Il est bien connu que la « loi de passage » (topos) est fréquemment omise, mais la réflexion doit sans doute s'ouvrir tant aux «prémisses manquantes » qu'à la possibilité d'une « conclusion absente » (Dufour $2008: 110-118$ ).

(iii) Enfin, une approche textuelle doit avancer dans la saisie de l'argumentation non seulement à l'échelle de la séquence (regroupement typé d'énoncés), mais aussi à l'échelle plus large du texte. Pour aller dans ce sens, il convient de se poser la question de la complexité : à partir du schéma de Toulmin et d'une modélisation de la séquence argumentative de base, quelles sont les structures plus complexes que l'on est amené à observer dans des réalisations textuelles effectives? La recherche anglophone s'intéresse depuis plusieurs années aux complex argumentation structures et envisage notamment trois cas de figure (voir Snoeck Henkemans 2003) : l'argumentation multiple (dans laquelle plusieurs prémisses soutiennent une conclusion de façon indépendante), coordonnée (dans laquelle plusieurs prémisses soutiennent la conclusion de façon interdépendante) et subordonnée (dans laquelle une prémisse soutient une conclusion qui, à son tour, devient une prémisse dans le cadre d'un nouveau mouvement argumentatif). La typologie de ces structures «complexes » est utile, mais on voit qu'elle ne saurait suffire à elle seule pour décrire l'argumentation à l'échelle d'un texte entier: c'est là un chantier encore vaste, auquel une approche comme la nôtre souhaite contribuer.

\section{De la gestion interactionnelle du désaccord au travail dialogique de positionnement}

Dans les sciences du langage francophones, l'intérêt pour la problématique séquentielle et textuelle de l'étayage a longtemps prédominé au point d'éclipser la dimension davantage relationnelle et pragmatique de l'argumentation, autrement dit l'expérience du désaccord et la confrontation de discours et contrediscours autour de questions communes. Grâce aux travaux de Plantin consacrés à la mise au point d'un modèle « dialogal » (voir par exemple 1996b, 2005), la dimension dialectique de l'argumentation a connu un regain d'intérêt ${ }^{13}$. C'est ce que nous avons proposé de saisir à travers la notion de positionnement, empruntée à Angenot : " [les humains] argumentent pour se situer par rapport aux raisons des autres en testant la cohérence et la force qu'ils imputent à leurs positions, pour se positionner [...]» (2008:441).

Il nous apparaît important de distinguer deux dimensions impliquées dans le positionnement argumentatif et selon lesquelles on peut envisager la relation qu'entretiennent discours et contrediscours : $(i)$ en tant que manière de co-agir et plus simplement d'être-ensemble, le désaccord - quel que soit pour le moment le contexte dans lequel il trouve à s'exprimer - définit la pertinence d'une approche fondamentalement interactionnelle de l'argumentation; (ii) chaque contribution argumentative - quelle que soit pour le moment la forme que prend cette contribution - témoigne d'un travail dialogique et passe par l'inscription et la prise en compte, par le locuteur, des arguments avancés par les autres agents engagés dans le désaccord. Ces deux niveaux, interactionnel et dialogique, font souvent l'objet d'une répartition étanche $d u$ travail, motivée essentiellement par les différences de type de données considérées. Tandis que les recherches consacrées aux données orales synchrones (pour le dire vite, les contextes « dialogaux ») se concentrent sur les enjeux interactionnels de l'argumentation et de la gestion des désaccords, les recherches qui se concentrent sur les données scripturales asynchrones (y compris les données scripturales oralisées telles que les allocutions) se focalisent sur l'inscription dialogique de l'argumentation de l'adversaire dans les textes monologalement argumentés. Notre intention est d'éviter de reconduire ces différences méthodologiques essentiellement imputables aux types de supports et de 
privilégier une analyse des procédures et des ressources transversales du positionnement argumentatif, tout en restant attentifs aux pressions exercées par les particularités des situations de communication.

(i) La dimension interactionnelle va de soi pour les recherches consacrées aux contextes argumentatifs dialogaux, où la construction du désaccord et des positions antagonistes se fonde sur l'alternance des tours de parole des différents locuteurs. Autant les discours et contre-discours que les rôles de Proposant et d'Opposant (suivant la terminologie de Plantin 2005) sont ainsi distribués entre les différents agents engagés dans un désaccord in praesentia ${ }^{14}$. Quand bien même l'analyse interactionnelle de l'argumentation est encore peu représentée dans le champ francophone, on peut citer les analyses linguistiques de la gestion interactionnelle de l'accord et du désaccord argumenté dans les débats médiatiques (Doury 1997; Sitri 2003 ; Burger 2005 ; Doury 2009 ; Doury \& Kerbrat-Orecchioni 2011), dans les débats publics (Jacquin 2011c, 2011b), dans les réunions professionnelles et les interactions de service (Traverso 2005) ou encore dans la conversation ordinaire (Traverso 1999, 2007 [1999]).

En quoi les contextes monologaux, c'est-à-dire les contextes de parole où les locuteurs ne peuvent s'interrompre entre l'ouverture et la clôture, sont-ils concernés par cette problématique interactionnelle? Les agents engagés dans le désaccord ne sont certes pas en situation de pouvoir le négocier on line, mais ce désaccord reste constitutif de leur relation et donne sens à leur argumentation respective. Ouvrir le texte monologal argumentatif sur ses dimensions interactionnelles, comme le propose Plantin (2005, 2012), constitue par conséquent un enjeu essentiel. Il faut entendre cette ouverture « interactionnelle » du texte monologal au sens fort et non au sens du seul « dialogisme », notion renvoyant au fait que tout texte inscrit une certaine altérité en son sein (voir infra). Par « dimension interactionnelle », il s'agit de rendre compte de la relation que les deux locuteurs à l'origine de deux textes monologaux distincts entretiennent l'un avec l'autre par le truchement de ces textes. Une telle proposition pose néanmoins des questions méthodologiques complexes, dont la principale a trait à la construction des corpus : comment construire un corpus de textes monologaux qui puisse non seulement rendre visibles les positions tenues par les différents protagonistes, mais également rendre compte de la gestion interactionnelle du désaccord qui les « réunit»? C'est une réflexion en cours. On peut trouver dans l'émergence d'une sociologie des controverses fondée sur l'analyse de grands corpus hétérogènes une possibilité de rendre à la fois manifeste et analysable la co-présence, au sein de l'espace public, de discours et de contre-discours instanciés dans des textes monologaux produits par des agents distincts (voir par exemple Chateauraynaud $2011^{15}$ ). Ce phénomène de co-présence peut néanmoins apparaître comme un artefact aux yeux de l'interactionniste strict, intéressé par la négociation située du désaccord. Sommes-nous face à un clivage théorique ? Tout le laisse croire, mais nous comptons défendre que l'analyse argumentative du texte monologal a tout à gagner à s'ouvrir vers l'interactionnel, ce qui, d'un point de vue méthodologique, « entraîne une révision de ce qu'est fondamentalement une donnée en argumentation (au moins une paire de discours en contradiction) »(Plantin 2005 : 73, voir également Doury 2004b).

(ii) Du côté de la dimension dialogique, on envisage le fait que le sujet argumentant construit sa position relativement à celle(s) des autres, c'est-à-dire qu'il la situe dans le désaccord. Il s'agit de prêter attention aux diverses ressources qui s'offrent au locuteur pour inscrire autrui (ses propos, sa position, son argumentation) dans sa propre parole. Ceci nous apparaît valable indépendamment du contexte considéré. Tout sujet argumentant est concerné et le fait que les locuteurs aient la possibilité ou non de s'interrompre pour contre-argumenter n'est pas pertinent.

Avec cette dimension dialogique, on rejoint la problématique de "l'hétérogénéité énonciative représentée » et plus spécifiquement de la "représentation du discours autre » (Authier-Revuz 1995, 2004) : que la parole de l'autre fasse l'objet d'une «prédication» (discours direct, discours indirect) ou d'une «modalisation» (discours second, modalisation autonymique), l'altérité est non seulement délimitée par des indicateurs de portée (voir aussi Charolles 1988), mais aussi attribuée à un responsable, vis-à-vis de l'argumentation duquel le sujet argumentant se positionne ${ }^{16}$. Outre ces phénomènes de représentation, on prendra également en compte la «polyphonie », c'est-à-dire les différentes ressources qui s'offrent au sujet argumentant pour inscrire non plus des discours, mais des points de vue (voir par exemple Ducrot 1984; Nølke 2001) $)^{17}$. Qu'il s'agisse de représentation du discours autre ou de polyphonie, on sera attentifs aux moyens permettant au sujet argumentant de prendre en compte 
l'argumentation d'autrui sans pour autant la prendre à sa charge (Coltier, Dendale \& De Brabanter 2009).

L'analyse de l'inscription du discours et du point de vue autre a été en premier lieu développée dans les recherches consacrées à l'étude des textes monologaux, avec l'idée de donner une assise linguistique à l'idée bakthinienne d'un dialogisme généralisé, d'une parole toujours traversée par la parole des autres, autrement dit encore du fait que tout texte, aussi monologal soit-il, constitue déjà une réponse (pour une synthèse sur ce type de travaux, voir par exemple le recueil publié par Bres, et al. 2005). Aujourd'hui communément admis, les instruments de l'analyse dialogique et polyphonique sont régulièrement exploités dans les analyses argumentatives de textes monologaux (voir par exemple Amossy 2005).

Du côté de l'analyse du dialogue, la prise en compte de la dimension dialogique est longtemps restée dans l'ombre du primat accordé à l'étude des dynamiques interactionnelles. Depuis quelques années, plusieurs travaux tentent de remédier à ce déficit, non sans pointer la complexité de la tâche. Dans le cas de données orales synchrones, la ligne de partage entre les phénomènes imputables au dialogal et au dialogique n'est en effet pas aisée à établir (Bres \& Nowakowska 2006, 2008). La réflexion est du moins en cours et plusieurs chercheurs se consacrent à l'analyse des enjeux interactionnels et argumentatifs de l'inscription du discours et du point de vue autre dans le dialogue (voir par exemple Vincent \& Heisler 1999 ; Doury 2004a ; Magaud 2004 ; Bres \& Nowakowska 2006, 2008 ; Rabatel 2009 ; Jacquin 2011b).

De notre point de vue, il s'agit donc de considérer que les dimensions interactionnelle et dialogique sont consubstantielles à l'argumentation et qu'elles se laissent embrasser par la problématique du positionnement. Ceci implique qu'une attention toute particulière soit portée aux zones laissées traditionnellement dans l'ombre des clivages méthodologiques imputables à une superposition, souvent reconduite, entre type de données et type d'approche : la dimension interactionnelle de l'argumentation dans le monologue et la dimension dialogique de l'argumentation dans le dialogue.

\section{Conclusion}

Notre contribution poursuivait deux objectifs. Nous voulions d'abord soumettre à la discussion une définition de travail de l'argumentation qui soit à la fois spécifiante et transversale. Spécifiante, parce qu'il s'agissait de se donner pour objet une forme localisable et observable, une pratique langagière particulière, et d'éviter ainsi sa dissolution dans la langue ou dans le discours. Transversale ensuite, parce qu'il s'agissait de localiser le fait argumentatif indépendamment du lieu générique ou du support de sa manifestation. Nous entendions proposer une saisie de « ce qui fait argumentation » dans la diversité des formes de son actualisation. Pour ce faire, nous avons convoqué une pluralité d'approches, allant des analyses interactionnelles aux analyses textuelles, de la problématique de la séquentialité à celle du dialogisme, faisant l'hypothèse que l'argumentation et la notion de « position » qui en constitue le cœur gagnent à être abordées au travers d'une diversité de prismes méthodologiques.

Le lecteur peut saisir en filigrane le second objectif de notre contribution. Il s'agissait d'utiliser l'argumentation comme un terrain d'investigation précieux pour les sciences du langage, à même de montrer l'utilité de dépasser les frontières disciplinaires découlant d'une répartition étanche des types de données pour approcher les pratiques langagières dans la complexité de leur rapport à la langue et au discours, d'entreprendre des démarches interdisciplinaires et collectives au sein même des sciences du langage.

\section{Références bibliographiques}

Adam, J.-M. (2004). Une approche textuelle de l'argumentation : "schéma", séquence et phrase périodique. In Doury, M. \& Moirand, S. (Eds.), L'argumentation aujourd'hui : positions théoriques en confrontation. Paris : Presses Sorbonne Nouvelle, 78-102.

Adam, J.-M. (2011). Les textes : types et prototypes. Paris : Armand Colin. 
Amossy, R. (2005). De l'apport d'une distinction : dialogisme vs polyphonie dans l'analyse argumentative. In Bres, J., Haillet, P. P., Mellet, S. et al. (Eds.), Dialogisme et polyphonie : approches linguistiques. Bruxelles : De Boeck Université, 63-73.

Amossy, R. (2010 [2000]). L'argumentation dans le discours. Paris : Armand Colin.

Angenot, M. (2008). Dialogues de sourds : traité de rhétorique anti-logique. Paris : Mille et une nuits.

Anscombre, J.-C. \& Ducrot, O. (1988). L'argumentation dans la langue. Bruxelles : Mardaga.

Aristote. (1991). Rhétorique. Paris : Gallimard.

Atayan, V. (2009). Fonctions argumentatives secondaires dans l'argumentation textuelle. In Atayan, V. \& Pirazzini, D. (Eds.), Argumentation : théorie - langue - discours. Frankfurt: Peter Lang, 93-110.

Authier-Revuz, J. (1995). Ces mots qui ne vont pas de soi. Boucles réflexives et non-cö̈ncidences du dire. Paris : Larousse.

Authier-Revuz, J. (2004). La représentation du discours autre : un champ multiplement hétérogène. In Munoz, J. M. L., Marnette, S. \& Rosier, L. (Eds.), Le discours rapporté dans tous ses états. Paris : L'Harmattan, 35-53.

Bres, J., Haillet, P. P., Mellet, S., et al. (Eds.). (2005). Dialogisme et polyphonie: approches linguistiques. Bruxelles : De Boeck Université.

Bres, J. \& Nowakowska, A. (2006). Dialogisme : du principe à la matérialité discursive. Recherches linguistiques, vol.28, 21-48.

Bres, J. \& Nowakowska, A. (2008). "J'exagère?... " Du dialogisme interlocutif. In Birkelund, M., Mosegaard Hansen, M.-B. \& Norén, C. (Eds.), L'énonciation dans tous ses états. Bruxelles : Peter Lang, 1-27.

Burger, M. (2005). La complexité argumentative d'une séquence de débat politique médiatique. In Burger, M. \& Martel, G. (Eds.), Argumentation et communication dans les médias. Québec : Nota Bene, 51-79.

Carel, M. (2011). L'entrelacement argumentatif-Lexique, discours et blocs sémantiques. Paris : Champion.

Charaudeau, P. (2009). L'argumentation dans une problématique d'influence. Argumentation et Analyse du Discours, vol., $n^{\circ} 1$, disponible à l'adresse http://aad.revues.org/193, consulté le 27 octobre 2011.

Charolles, M. (1988). Les plans d'organisation textuelle : périodes, chaînes, portées et séquences. Pratiques, $n^{\circ} 57$, $3-13$.

Chateauraynaud, F. (2011). Argumenter dans un champ de forces. Essai de balistique sociologique. Paris : PETRA.

Coltier, D., Dendale, P. \& De Brabanter, P. (2009). La notion de prise en charge : mise en perspective. Langue française, vol.2, $n^{\circ} 162,3-27$.

Doury, M. (1997). Le débat immobile. L'argumentation dans le débat médiatique sur les parasciences. Paris : Kimé.

Doury, M. (2003). L'évaluation des arguments dans les discours ordinaires. Le cas de l'accusation d'amalgame. Langage et société, vol.3, $n^{\circ} 105,9-37$.

Doury, M. (2004a). La fonction argumentative des échanges rapportés. In Munoz, J. M. L., Marnette, S. \& Rosier, L. (Eds.), Le discours rapporté dans tous ses états. Paris : L'Harmattan, 254-264.

Doury, M. (2004b). La position du chercheur en argumentation. Semen, $n^{\circ} 17,149-163$.

Doury, M. (2009). "Sans faire allégeance" : l'euphémisme dans l'expression des accords et désaccords dans un débat politique. Synergies Italie, $n^{\circ}$ spécial, 111-124.

Doury, M. \& Kerbrat-Orecchioni, C. (2011). La place de l'accord dans l'argumentation polémique : le cas du débat Sarkozy/Royal (2007). A contrario, $n^{\circ} 16,63-87$

Ducrot, O. (1984). Le dire et le dit. Paris : Minuit.

Ducrot, O. (2004). Argumentation rhétorique et argumentation linguistique. In Doury, M. \& Moirand, S. (Eds.), L'argumentation aujourd'hui : positions théoriques en confrontation. Paris : Presses Sorbonne Nouvelle, 17-34.

Dufour, M. (2008). Argumenter : cours de logique informelle. Paris : Armand Colin.

Govier, T. (1997). A practical study of argument. Belmont: Wadsworth Publishing Company. 
Grize, J.-B. (1982). De la logique naturelle à l'argumentation. Genève : Droz.

Grize, J.-B. (1996). Logique naturelle et communications. Paris : PUF.

Jacquin, J. (2011a). Le/La polémique : une catégorie opératoire pour une analyse discursive et interactionnelle des débats publics? Semen, $n^{\circ} 31,43-60$.

Jacquin, J. (2011b). Localiser, décrire et faire voir le fait argumentatif : le modèle dialogal de l'argumentation au défi d'un corpus complexe. A contrario, $n^{\circ} 16,88-109$.

Jacquin, J. (2011c). Orientation interactionnelle et multimodale vers l'activité de "débattre". Analyse d'un extrait de débat public. Mots, $n^{\circ} 96,45-62$.

Johnson, R. H. (2000). Manifest Rationality. Mahwah: Lawrence Erlbaum Associates.

Magaud, V. (2004). Discours rapporté et argumentation : l'exemple de la consultation de voyance radiophonique. In Munoz, J. M. L., Marnette, S. \& Rosier, L. (Eds.), Le discours rapporté dans tous ses états. Paris : L'Harmattan, 275-286.

Micheli, R. (2009). La construction de l'objet dans quelques approches discursives et interactionnelles de l'argumentation : une perspective métathéorique. In Atayan, V. \& Pirazzini, D. (Eds.), Argumentation : théorielangue-discours. Frankfurt: Peter Lang, 19-30.

Micheli, R. (2010). L'émotion argumentée. L'abolition de la peine de mort dans le débat parlementaire français. Paris : Le Cerf.

Micheli, R. (Ed.) (2011). L'argumentation au carrefour des disciplines : sciences du langage et sciences sociales, numéro spécial de la revue A Contrario, [en ligne], http://www.cairn.info/revue-a-contrario-2011-2.htm\#fiche.

Micheli, R. (2012). Arguing without Seeking to Persuade ? Elements for a Non Persuasive Conception of Argumentation. Argumentation. An International Journal on Reasoning, vol.26, $n^{\circ} 1,115-126$.

Nølke, H. (2001). Le regard du locuteur : pour une linguistique des traces énonciatives. Paris : Kimé.

Perelman, C. \& Olbrechts-Tyteca, L. (1970). Traité de l'argumentation. La Nouvelle Rhétorique. Bruxelles : Editions de l'Université de Bruxelles.

Plantin, C. (1990). Essais sur l'argumentation : introduction à l'étude linguistique de la parole argumentative. Paris : Kimé.

Plantin, C. (1996a). L'argumentation. Paris : Seuil.

Plantin, C. (1996b). Le trilogue argumentatif. Présentation de modèle, analyse de cas. Langue française, $n^{\circ} 112$, 9-30.

Plantin, C. (2003). Des polémistes aux polémiqueurs. In Declercq, G., Murat, M. \& Dangel, J. (Eds.), La parole polémique. Paris : Champion, 377-408.

Plantin, C. (2005). L'argumentation : histoire, théorie et perspectives. Paris : PUF.

Plantin, C. (2012). Persuasion or Alignment? Argumentation. An International Journal on Reasoning, vol.26, $n^{\circ} 1$, 83-97.

Rabatel, A. (2009). Prise en charge et imputation, ou la prise en charge à responsabilité limitée... Langue française, vol. $2, n^{\circ} 162,71-87$.

Sitri, F. (2003). L'objet du débat : la construction des objets de discours dans des situations argumentatives orales. Paris : Presses Sorbonne Nouvelle.

Snoeck Henkemans, F. (2003). Complex Argumentation in a Critical Discussion. Argumentation, vol.17, $n^{\circ} 4$, 405419.

Toulmin, S. E. (1958). The Uses of Argument. Cambridge : Cambridge University Press.

Traverso, V. (1999). Negociaciôn y argumentaciôn en la conversaciôn familiar. Escritos, $n^{\circ}$ spécial, 51-89.

Traverso, V. (2005). Cristallisation des désaccords et mise en place de négociations dans l'interaction : des variations situationnelles. In Grosjean, M. \& Mondada, L. (Eds.), Des négociations au travail. Lyon : PUL, 43-69.

Traverso, V. (2007 [1999]). L'analyse des conversations. Paris : Armand Colin. 
Van Eemeren, F. H. \& Grootendorst, R. (2004). A Systematic Theory of Argumentation : the pragma-dialectical approach. Cambridge : Cambridge University Press.

Vincent, D. \& Heisler, T. (1999). L'anticipation d'objections : prolepse, concession et réfutation dans la langue spontanée. Revue québécoise de linguistique, vol.27, $n^{\circ} 1,15-31$.

Walton, D., Reed, C. \& Macagno, F. (2008). Argumentation Schemes. Cambridge : Cambridge University Press.

\begin{abstract}
${ }^{1}$ Raphaël Micheli s'intéresse aux rapports qui, dans le débat politique, se nouent entre la sémiotisation verbale des émotions et le fonctionnement de l'argumentation (Micheli 2010). Sur un plan méthodologique plus général, il aborde le problème de la définition de la notion d'" argumentation » en sciences du langage, notamment dans une optique discursive (Micheli 2009, 2011) : il tente, à ce sujet, de contribuer à l'élaboration d'une définition de l'argumentation qui ne repose pas - à la différence de ce qu'enseigne la tradition rhétorique - sur la visée persuasive du locuteur (Micheli 2012). Jérôme Jacquin s'intéresse à la pratique de l'argumentation et aux modes de sémiotisation de l'identité dans des contextes oraux synchrones de type «débat public» (Jacquin 2011c, 2011b). Ses recherches participent à l'identification des structures de l'argumentation telles qu'elles se manifestent dans l'interaction et à celle de formes connexes de confrontation verbale, comme la polémique (Jacquin 2011a).
\end{abstract}

${ }^{2}$ Nous prenons ici en considération des orientations méthodologiques qui se développent dans le champ des sciences du langage contemporaines et qui - à divers titres - s'intéressent à la description de l'argumentation. Il est clair, par ailleurs, que ces orientations se nourrissent de traditions bien plus anciennes, au premier rang desquelles figure certainement la rhétorique. L'influence de celle-ci est en effet très perceptible dans certains modèles discursifs actuels de l'argumentation: on pense ici en premier lieu à la théorie de l'« argumentation dans le discours » (Amossy 2010 [2000]), qui sera discutée plus loin.

${ }^{3}$ L'importance de ces deux composants a été régulièrement soulignée par les chercheurs effectuant un travail métathéorique sur la notion d'argumentation. La présentation simplifiée que l'on en propose ici ne doit pas laisser croire qu'ils constituent les termes d'une dichotomie, ou qu'il s'agit là des deux seuls critères pertinents pour définir cette notion. Notre hypothèse est plutôt que ces deux composants exercent une fonction véritablement structurante dans les débats méthodologiques sur l'étude de l'argumentation en sciences du langage : lorsqu'elle avance une définition de l'argumentation, chaque théorie opère à sa manière un travail - parfois implicite - de pondération de ces composants.

${ }^{4}$ Le choix du genre de la «définition» ne doit pas être mal compris. Il ne s'agit pas d'adopter une quelconque posture doctrinaire : nous n'avons bien sûr pas la prétention d'imposer une définition, dans le but unique qu'elle soit ratifiée et appliquée par d'autres chercheurs. Une définition constitue à nos yeux non pas un geste d'autorité par lequel on se soustrait au débat avec ses pairs, mais bien, à l'inverse, un moyen privilégié - parce que direct-de participer à ce débat.

${ }^{5}$ Il faut préciser d'emblée que l'intérêt potentiel de la définition avancée réside selon nous dans la conjonction des différents critères retenus. Pris isolément, aucun de ces critères ne suffit à saisir ce que l'argumentation peut avoir de spécifique en tant que mode d'organisation du discours. Il existe notamment - nous y reviendrons - d'autres formes de traitement verbal du désaccord (critère 1). Par ailleurs, lorsqu'il est considéré en soi, le travail interactionnel et dialogique de positionnement (critère 5) peut être jugé inhérent à toute activité énonciative (qu'elle relève ou non de l'argumentation).

${ }^{6}$ Notamment par Plantin (1996a: 18) : «Si on considère l'argumentation comme un fait de discours, [...] deux options sont possibles : - Toute parole est nécessairement argumentative. C'est un résultat concret de l'énonciation en situation. Tout énoncé vise à agir sur son destinataire [...] et à transformer son système de pensée. - Certains discours seulement sont argumentatifs. L'argumentativité est à rechercher dans le mode d'organisation des discours »; voir aussi Amossy (2010 [2000] : 32-34).

${ }^{7}$ Plantin a souvent critiqué les diverses versions de l'argumentativisme généralisé, en parlant notamment du risque de « dissolution » de l'argumentation dans le « sens, le langage, l'information » (Plantin 2005 : 34).

${ }^{8}$ Plantin suggère à ce sujet que tant les approches rhétoriques que les approches normatives inspirées de la dialectique sont « orientées vers la recherche d'un consensus, capable de clore le débat » (2003:378-379)

${ }^{9}$ Idée souvent formulée par les théories, qui opposent l'argumentation à d'autres modes de traitement du désaccord, qu'ils soient verbaux ou non.

${ }^{10}$ Selon l'heureuse expression de Traverso (2007 [1999] : 76), reprise par Doury (2004a). 
11 «[Le sujet qui veut argumenter] doit se positionner par rapport à la problématisation proposée, dire quel est son point de vue par rapport aux assertions en présence. Il s'engage alors dans une prise de position en défendant l'une des deux assertions, ce qui le conduira du même coup à s'opposer à l'autre » (Charaudeau 2009).

${ }^{12}$ L'argumentation apparaît alors comme « un mode de construction du discours visant à le rendre plus résistant à la contestation » (Doury 2003 : 13 ; voir aussi Doury 2004a : 255 ; Plantin 2005 : 58-62).

${ }^{13}$ Parallèlement et depuis plusieurs années, on rencontre dans les sciences du langage un intérêt grandissant non seulement pour l'analyse des interactions en co-présence, mais plus généralement pour la problématique de l'inscription d'autrui dans le monologue (voir infra).

${ }^{14}$ On ne saurait toutefois aller trop vite en besogne. Dans le cas des débats médiatiques (par exemple les débats présidentiels français de l'entre-deux-tours), la gestion du désaccord passe bel et bien par la répartition de positions antagonistes entre les débattants engagés dans la confrontation. Néanmoins, on peut facilement envisager des contextes dialogaux (par exemple le brainstorming d'entreprise, ou la réunion de campagne électorale) où les locuteurs ne se répartissent pas en positions antagonistes, mais collaborent - et à la limite s'engagent dans des désaccords internes à leur position - pour construire une position argumentative commune à opposer à celle tenue par un tiers absent. Il serait alors particulièrement pertinent d'étudier la manière dont les locuteurs usent des ressources dialogiques dont il est question infra pour faire référence au discours argumentatif étayant la position non représentée in situ.

15 «Le prototype de la mise en tension des forces et des arguments a longtemps été incarné par le face-à-face des champions politiques lors des campagnes électorales. Mais les épreuves de vérité se déploient dans de multiples cadres, des plus formalisés aux situations de la vie ordinaire » (Chateauraynaud 2011 : 15).

${ }^{16}$ L'« hétérogénéité représentée », caractérisée par une opération de délimitation et d'attribution, se distingue de l' "hétérogénéité constitutive », "dégagé[e] et théorisé[e] dans des approches non proprement linguistiques du langage, reconnaissant [...] le caractère constitutif pour tout dire, et en chaque point, de l'ailleurs du déjà-dit» (Authier-Revuz 2004 : 51). Il s'agit de cet espace langagier interindividuel que tout énoncé mobilise, le plus souvent inconsciemment, sans le délimiter ni l'attribuer, et que d'autres circonscrivent par les notions de "dialogisme constitutif » et de « dialogisme interdiscursif » (voir par exemple Bres \& Nowakowska 2006).

${ }^{17}$ Voir par exemple le cas de la négation polémique, qui permet au locuteur d'invoquer un point de vue véhiculé par la forme positive tout en assumant le contenu inverse (Anscombre \& Ducrot 1988). 